\title{
Pain flare, complexity and analgesia in bone oligometastases treated with stereotactic body radiation therapy
}

\author{
Mauro Loi (i) | Natalie D. Klass | Kim C. De Vries | Emmanuelle Fleury | \\ Marieke Van Zwienen | Ilse de Pree | Joost Nuyttens
}

Department of Radiotherapy, Erasmus MC, Rotterdam, The Netherlands

Correspondence

Mauro Loi, Department of Radiation

Oncology, Erasmus MC Cancer Institute,

Rotterdam, The Netherlands.

Email:m.loi@erasmusmc.nl

Funding information

Swiss Cancer League

\begin{abstract}
The aim of our study was to assess the incidence of pain flare and the effectiveness of stereotactic body radiotherapy (SBRT) in pain management of patients with bone oligometastases. We evaluated 48 patients accounting for 54 treatments. The Edmonton Classification System for Cancer Pain (ECS-CP) was applied to identify indicators of treatment-resistant pain, in patients with active pain (NRS $\geq 2$ ) at baseline. Statistical analysis was performed to identify predictors of pain flare and pain control. Pain flare occurred in $38 \%$ of treated patients $(n=18 / 48)$ : No correlation was found between pain flare and patient- or treatment-related variables. In the subset of patients with active pain at baseline $(n=23)$, pain control was obtained in $62 \%$ of patients at 1 year; median time to pain progression after SBRT was 29 months (C195\% 6-52 months). Presence of $\geq 2$ ECS-CP features was correlated with earlier pain progression (4 vs. 30 months, $p=0.012$ ). Pain flare occurred in $38 \%$ of cases irrespectively of steroid premedication and dose regimen. In patient with baseline active pain, durable pain control was obtained. Presence of $\geq 2$ complexity indicators at the ECS-CP assessment was correlated with impaired pain control and may deserve future investigation in prospective studies.
\end{abstract}

\section{KEYWORDS}

bone metastases, pain control, pain flare, palliative care, SBRT

\section{1 | INTRODUCTION}

Bone metastases are a frequent occurrence in cancer patients, developing in one third of cases and resulting in pain associated with considerable morbidity (Van Oorschot, Rades, Schulze, Beckmann, \& Feyer, 2011). Traditional management of symptomatic bone lesion encompass surgery in selected patients, pharmacological interventions (pain medication, bisphosphonates) and conventional fractionated radiotherapy (CRT) to painful sites (Chow et al., 2012).

In recent years, use of stereotactic body radiotherapy (SBRT), a highly focused ablative radiotherapy technique, has been reported by multiple institutions for the treatment of bone metastases, showing promising results in terms of local control, pain relief, safety and cost-effectiveness (Bhattacharya \& Hoskin, 2015) though no direct comparison is available with CRT (De Bari et al., 2016). This is of primary interest in patients with limited disease burden, consisting of $\leq 3$ concurrent metastases, who experience longer lifespan compared to patients with extensively disseminated disease (Jacobson, Shapiro, Abbeele, \& Kaplan, 2001). In this subset of metastatic patients, defined as "oligometastatic," the achievement of long-lasting analgesia and local control is particularly desirable. In a recent multi-institutional retrospective cohort, long-term disease control and survival were obtained following ablative radiotherapy for oligometastatic patients: most notably, bone metastatic involvement was not correlated with impaired efficacy, while a dose correlation with local control was seen in this subset of patients (Hong et al., 2018).

However, cancer pain is a complex clinical entity: Due to heterogeneity in the population of patients affected by painful bone 
metastases, it is of primary concern to identify patients who can benefit most from this approach and to assess its role in pain management. Multiple tools are available to further characterise chronic pain in cancer patients, in order to provide elements for clinical decision, but their use in the radiotherapy setting is limited. Among them, the Edmonton Classification System for Cancer Pain (ECS-CP) proved effective in predicting resistance to pain treatment according to the presence of five complexity features (neuroceptive component, incident pain, psychological distress, addictive behaviour and cognitive impairment; Fainsinger et al., 2005).

SBRT has common radiotherapy-related toxicities: In particular pain flare, a temporary increase in pain at the treated site (De Bari et al., 2016). Pain flare, despite its transient nature, is perceived as a debilitating and worrisome event by the majority of patients, with important drawbacks on daily activities and insufficient relief from increased pain medication (Hird et al., 2009). So, SBRT is a doubleedged sword: On the one hand, it is used as a long-lasting pain killer but on the other hand it can cause a pain flare. The aims of the study were to evaluate the variations in pain following stereotactic radiotherapy to bone metastases (spinal and extraspinal). We assessed the incidence of pain flare following the first treatment fraction and the pain control after the treatment and their respective predictive factors.

\section{2 | MATERIALS AND METHODS}

\section{1 | Patient selection, procedures and follow-up}

A retrospective review of oligometastatic ( $\leq 3$ concurrent metastases) patients treated at our Institution between May 2005 and September 2016 with stereotactic radiotherapy for at least one bone metastasis was performed after obtainment of informed consent. Pain at the treated site was evaluated at baseline and during clinical follow-up after treatment using the Numeric Rating Scale (NRS) and Visual Analog Scale (VAS; Paice \& Cohen, 1997). In patient with NRS $\geq 2$ at baseline, active pain was further classified using the ECS-CP 5 items tool. Pain medications were classified according to the WHO three-step ladder, consisting of step 1 drugs (paracetamol, NSAIDs), step 2 drugs (mild opioids: codeine, dihydrocodeine or tramadol) and step 3 drugs (strong opioids: morphine, fentanyl, buprenorphine, oxymorphone, oxycodone or hydromorphone; WHO's Pain Ladder, 1986). Stereotactic treatment was delivered using the CyberKnife radiotherapy system (Accuray Inc, Sunnyvale, CA). Volumes delineation and dose calculation were performed using the Multiplan Treatment Planning System. The gross tumour volume (GTV) was identified as the visible lesion on fused diagnostic imaging (MRI, contrast-enhanced CT or PETCT). Planning target volume (PTV) included the GTV or, if present, the CTV plus a 2-5 mm margin. Before 2012, the PTV for spinal metastases equalled to the GTV plus a 2-3 mm margin, and after 2012, volumes were delineated according to the ISRC consensus recommendations (Cox et al., 2012). The total dose was prescribed to the outer line of the PTV to the $80 \%-90 \%$ isodose line using different single or multiple fraction schedules. Dose constraints for OARs were applied according to the American Association of Physicists in Medicine (AAPM) recommendations (Benedict et al., 2010). To take into account different dose fractionation, all the dosimetric variables were recalculated as equivalent dose in $2 \mathrm{~Gy}$ (EQD2) with an $\alpha / \beta=10$ and 3 for early and late reacting tissues respectively. For every treatment, patient-related data (age, gender, primary tumour, ECOG performance status, prior chemotherapy, number of metastases, spinal/extraspinal localisation, lytic/solid/ mixed radiological aspect, pain at baseline, use and dosage of analgesics, use and dosage of steroids) and radiotherapy-related data (single/multiple fraction dose schedule, total dose delivered, GTV and PTV volume, minimum dose to the PTV, maximum dose to the PTV, mean dose to the PTV) were collected. Likewise, opioids and steroid dosage (in milligrams) were reconverted in oral-morphine equivalent (OME) and dexamethasone equivalent (DXE) to allow for comparison. According to institutional guidelines, follow-up visits were scheduled at 1, 3 and 6 months and subsequently once a year. All procedures in our study were performed in accordance with the ethical standards of the National Research Committee and with the 1964 Helsinki declaration and its later amendments or comparable ethical standards.

\section{2 | Definition of endpoints}

Pain flare was defined as a temporary (resolving within subsequent clinical evaluation) $\geq 2$-points increase in the NRS pain scale compared to baseline levels with no decrease in analgesic intake or $+25 \%$ increase in analgesic intake with no decrease in pain score (Chow, Ling, Davis, Panzarella, \& Danjoux, 2005).

Pain progression (PP) was defined as durable increase of pain score of $\geq 2$ points above baseline at the treated metastatic site with stable analgesic use or increase $\geq 25 \%$ in daily oral-morphine equivalent compared with pretreatment intake, with stable pain score (Chow et al., 2005). Pain control (PC) was defined as time from treatment start date to PP. Overall survival (OS) was measured from start of the radiation therapy until death from any cause.

\section{3 | Statistical analysis}

Descriptive statistics were used to report patient- and treatment-related characteristics as mean and median with range for continuous variables and as proportions for categorical variables. To search for the relationship between pain flare and predictive factors, continuous parametric and non-parametric variables were tested with $t$ test and Mann-Whitney test, respectively, while chi-square test was used for categorical variables. Time-related events such as PC and OS were plotted using the Kaplan-Meier method; correlation with pretreatment was tested using the log-rank test. A $p$-value $\leq 0.05$ was considered statistically significant. Statistical analysis was performed with IBM SPSS v.21 statistical software. 
TAB LE 1 Baseline patient-related characteristics (per patient and per treatment site)

\begin{tabular}{|c|c|c|c|c|}
\hline & $\begin{array}{l}\text { Per patient } \\
\text { (total = 48) }\end{array}$ & $\%$ & $\begin{array}{l}\text { Per treatment } \\
\text { site }(\text { total }=54)\end{array}$ & $\%$ \\
\hline \multicolumn{5}{|l|}{ Gender } \\
\hline Men & 22 & 46 & 24 & 45 \\
\hline Women & 26 & 54 & 30 & 55 \\
\hline \multicolumn{5}{|c|}{ ECOG performance status } \\
\hline$\leq 1$ & 43 & 90 & 48 & 89 \\
\hline$>1$ & 5 & 10 & 6 & 11 \\
\hline \multicolumn{5}{|c|}{ Age $($ median $=65)$} \\
\hline$\leq 65$ years & 31 & 65 & 34 & 63 \\
\hline$>65$ years & 17 & 35 & 20 & 37 \\
\hline \multicolumn{5}{|l|}{ Primary tumour } \\
\hline Breast & 17 & 35 & 21 & 39 \\
\hline Colorectal & 4 & 8 & 4 & 7 \\
\hline Prostate & 9 & 19 & 10 & 18 \\
\hline $\begin{array}{l}\text { Head and } \\
\text { neck }\end{array}$ & 1 & 2 & 1 & 2 \\
\hline $\begin{array}{l}\text { Non-small- } \\
\text { cell lung } \\
\text { cancer }\end{array}$ & 10 & 20 & 10 & 18 \\
\hline Stomach & 3 & 6 & 3 & 6 \\
\hline Thyroid & 2 & 5 & 2 & 4 \\
\hline Kidney & 2 & 5 & 3 & 6 \\
\hline \multicolumn{5}{|c|}{ Prior chemotherapy } \\
\hline Yes & 19 & 40 & 21 & 39 \\
\hline No & 29 & 60 & 33 & 61 \\
\hline \multicolumn{5}{|c|}{ Number of concurrent metastases } \\
\hline 1 & 36 & 75 & 36 & 67 \\
\hline$\geq 2$ & 12 & 25 & 18 & 33 \\
\hline \multicolumn{5}{|c|}{ Radiological aspect } \\
\hline Lytic & N/A & - & 28 & 52 \\
\hline Solid & & - & 17 & 31 \\
\hline Mixed & & - & 9 & 17 \\
\hline \multicolumn{5}{|c|}{ Site of metastases } \\
\hline Spinal & N/A & - & 32 & 59 \\
\hline Extraspinal & & - & 22 & 41 \\
\hline \multicolumn{5}{|c|}{ Active pain at baseline (NRS $\geq 2$ ) } \\
\hline No & 25 & 52 & 31 & 57 \\
\hline Yes & 23 & 48 & 23 & 43 \\
\hline \multicolumn{5}{|l|}{ Pain medication } \\
\hline No & 25 & 60 & 31 & 57 \\
\hline WHO Step 1 & 7 & 13 & 9 & 17 \\
\hline WHO Step 2 & 3 & 5 & 3 & 6 \\
\hline WHO Step 3 & 11 & 22 & 11 & 20 \\
\hline \multicolumn{5}{|l|}{ Opioids } \\
\hline No opioids & 37 & 77 & 43 & 80 \\
\hline
\end{tabular}

TABLE 1 (Continued)

\begin{tabular}{|ccccc|}
\hline & $\begin{array}{c}\text { Per patient } \\
\text { (total = 48) }\end{array}$ & $\%$ & $\begin{array}{l}\text { Per treatment } \\
\text { site (total = 54) }\end{array}$ & $\%$ \\
\hline $\begin{array}{c}\text { <40 mg } \\
\text { OME }\end{array}$ & 6 & 13 & 6 & 11 \\
\hline $\begin{array}{c}\text { > } 0 \mathrm{mg} \\
\text { OME }\end{array}$ & 5 & 10 & 5 & 9 \\
\hline $\begin{array}{c}\text { Steroids } \\
\text { No steroids }\end{array}$ & 38 & 80 & 42 & \\
\hline$<8$ mg DXE & 5 & 10 & 7 & 78 \\
\hline$\geq 8$ mg DXE & 5 & 10 & 5 & 13 \\
\hline
\end{tabular}

Note. DXE: dexamethasone equivalent (in mg); OME: Oral-morphine equivalent (in $\mathrm{mg}$ ).

\section{3 | RESULTS}

\section{1 | Patient- and treatment-related characteristics}

A total number of 48 patients with 54 lesions were included in the study. The most represented primary tumour was breast adenocarcinoma, accounting for $21 / 54$ metastases (39\%) in $17 / 48$ patients (35\%). Bone was the only metastatic site in 36 patients out of 48 (75\%); 6 patients simultaneously received SBRT to two bone metastases. Bone metastases were predominantly located in the spine ( $n=32,59 \%)$, the pelvis ( $n=10,19 \%)$, the ribs $(n=7,13 \%)$, the limbs ( $n=4,7 \%)$ and in the skull $(n=1,2 \%)$. A pain level equal or superior to 2 in the NRS scale was present in 23 out of 48 patients, (48\%) all of whom treated for a single lesion. Daily intake of pain medications was found in 21 painful patients, consisting of step 1, step 2 and step 3 drugs in 7, 3 and 11 patients respectively. In patients requiring step 3 medication, median prescribed dose was $40 \mathrm{mg}$ OME (5-460 mg OME). Prescription of prophylactic steroid treatment was found in 10 patients, receiving a median dose of $8 \mathrm{mg}$ DXE (3-16 mg DXE). Use of steroids was more frequent in patients with concurrent opioid-based pain medication ( $p=0.02$ ). Patient-related characteristics are reported in Table 1.

Presence of pain complexity indicators was evaluated according to the ECS-CP tool in 23 patients with active pain before treatment (Table 2). Presence of incident pain was the most prominent ECS-CP feature (35\%), while no cognitive dysfunction was found in our cohort. At least one item was found positive in 13 patients, while five patients showed two or more ECS-CP features. No item or combination of items was correlated with administration of steroids or opioids, except for psychological distress that was correlated with higher steroid intake ( $p=0.008)$.

Different dose regimens were used, ranging from 20 to $48 \mathrm{~Gy}$ in 1-6 fractions (dose per fraction 7-20), corresponding to a median EQD2 prescribed dose of $44.0 \mathrm{~Gy}_{10}$ (29.8-72.0). The use of a single fraction of $20 \mathrm{~Gy}$ was restricted to nine spine metastases. The most used schedule was $24 \mathrm{~Gy}$ in two fractions, that was used in 34 of the 52 treatments. Treatment-related data are shown in Table 3. An SBRT planning case is provided as 


\begin{tabular}{|c|c|c|c|c|c|}
\hline & $N($ total $=23)$ & $\%$ & $\begin{array}{l}\text { Steroids (U } \\
\text { test) }\end{array}$ & $\begin{array}{l}\text { Opioids (U } \\
\text { test) }\end{array}$ & $\begin{array}{l}\text { Pain control } \\
\text { (log-rank test) }\end{array}$ \\
\hline \multicolumn{6}{|c|}{ Neuropathic pain } \\
\hline No & 17 & 74 & NS & NS & NS \\
\hline Yes & 6 & 26 & & & \\
\hline \multicolumn{6}{|c|}{ Incident pain } \\
\hline No & 15 & 65 & NS & NS & NS \\
\hline Yes & 8 & 35 & & & \\
\hline \multicolumn{6}{|c|}{ Psychological distress } \\
\hline No & 19 & 83 & $p=0.008$ & NS & NS \\
\hline Yes & 4 & 17 & & & \\
\hline \multicolumn{6}{|c|}{ Addictive behaviour } \\
\hline No & 22 & 96 & NS & NS & NS \\
\hline Yes & 1 & 4 & & & \\
\hline \multicolumn{6}{|c|}{ Cognitive dysfunction } \\
\hline No & 23 & 100 & - & - & - \\
\hline Yes & 0 & 0 & & & \\
\hline \multicolumn{6}{|c|}{ ECS-CP indicators } \\
\hline 0 & 10 & 44 & - & - & - \\
\hline$\geq 1$ & 13 & 56 & NS & NS & NS \\
\hline$\geq 2$ & 5 & 22 & NS & NS & $p=0.012$ \\
\hline
\end{tabular}

TABLE 2 Pain complexity according to ECS-CP (per patient and per treatment site) and correlation with steroid use, opioid medication and pain progression in patients with active pain at baseline

Data in bold represents values that are statistically significant $(p<0.05)$ at statistical analysis.

Figure S1. The size of the GTV was correlated at univariate analysis with active pain in the treated site $(p<0.001)$ and consequently with prescription of pain medication $(p<0.001)$ and in particular opioids $(p=0.005)$.

\section{2 | Pain flare}

Following SBRT, pain flare was reported at the first follow-up visit in 18 treated sites, accounting for $34 \%$ of treatments in 18 (38\%) patients. No other acute side effects were observed in our cohort. Eleven patients experienced pain flare as an exacerbation of pre-existing active pain. Pain flare was observed in 5 of the 10 patients (50\%) receiving prophylactic steroid treatment and in 6 out of the 14 patients (43\%) receiving a weak to strong opioid medication (5/11 and $1 / 3$ for WHO grade 2 and 3 medication respectively). Concerning tumour localisation, pain flare occurred in $38 \%$ of spinal metastases and in $28 \%$ of extraspinal metastases (12/32 and 6/22 respectively). Pain flare was present in $45 \%$ of single-fraction treatments $(4 / 9)$ and in $31 \%(14 / 45)$ of multiple fraction treatments. At univariate analysis, neither patient- nor treatment-related characteristics proved significantly correlated with the onset of pain flare. Pain flare regressed spontaneously $(n=9,17 \%)$ or after a transient increase in pain medication $(n=9,17 \%)$ with return to baseline intake at the time of the first follow-up visit at 1 month.

\section{3 | Pain control}

Pain control was obtained in 14/23 patients with baseline active pain, while no pain progression occurred in any of the previously asymptomatic patients. In two patients, surgical rescue due to radiologically assessed tumour progression was needed. By plotting pain progression in function of time with the Kaplan-Meier method (Figure 1, left), PC was $69 \%$ at 6 months and $62 \%$ at 1 year, with a median time to pain progression of 29 months (CI95\% 6-52 months). At univariate analysis, only the presence of at least two ECS-CP features was correlated with impaired PC (median PC 4 vs. 30 months, $p=0.012$; Figure 1, right). Median follow-up was 7 months (1-71 months). Median overall survival was 39 months (C195\% 15-64 months), resulting in a 6-month OS of $88 \%$ and a 1-year OS of $75 \%$ (Figure 2). Only breast as primary tumour $(p=0.019)$ and absence of steroid pretreatment $(p=0.045)$ were correlated with longer OS, though none of them proved significant at multivariate analysis.

\section{4 | DISCUSSION}

Pain flare was a frequent side effect of SBRT and was found in more than one third of patients in our series. The occurrence of pain flare is a common acute adverse effect in patients treated with CRT palliative dose schedules, with reported incidence up to $40 \%$ (Chow et al., 2005; Loblaw, Wu, Panzarella, \& T, Smith K, Aslanidis J, Warde P, 2007) as confirmed by a recent multi-institutional prospective trial 
TAB LE 3 Treatment-related characteristics (per treatment site)

\begin{tabular}{|c|c|c|}
\hline & $N($ total $=54)$ & $\%$ \\
\hline \multicolumn{3}{|l|}{ Dose Fractionation } \\
\hline Single fraction & 9 & 17 \\
\hline Multiple fractions & 45 & 83 \\
\hline \multicolumn{3}{|l|}{ GTV in $\mathrm{cm}^{3}$} \\
\hline$\leq 33$ & 39 & 72 \\
\hline$>33$ & 15 & 28 \\
\hline \multicolumn{3}{|l|}{ PTV in $\mathrm{cm}^{3}$} \\
\hline$\leq 64$ & 34 & 63 \\
\hline$>64$ & 20 & 37 \\
\hline \multicolumn{3}{|c|}{ Total dose prescribed in $\mathrm{Gy}_{10}$} \\
\hline$\leq 44$ & 36 & 67 \\
\hline$>44$ & 18 & 33 \\
\hline \multicolumn{3}{|c|}{ Maximum dose to PTV in $\mathrm{Gy}_{10}$} \\
\hline$\leq 62$ & 27 & 50 \\
\hline$>62$ & 27 & 50 \\
\hline \multicolumn{3}{|c|}{ Mean dose to PTV in $\mathrm{Gy}_{10}$} \\
\hline$\leq 48$ & 28 & 52 \\
\hline$>48$ & 26 & 48 \\
\hline \multicolumn{3}{|c|}{ Total dose prescribed in $\mathrm{Gy}_{3}$} \\
\hline$\leq 72$ & 36 & 67 \\
\hline$>72$ & 18 & 33 \\
\hline \multicolumn{3}{|c|}{ Maximum dose to PTV in $\mathrm{Gy}_{3}$} \\
\hline$\leq 107$ & 27 & 50 \\
\hline$>107$ & 27 & 50 \\
\hline \multicolumn{3}{|c|}{ Mean dose to PTV in $\mathrm{Gy}_{3}$} \\
\hline$\leq 82$ & 28 & 52 \\
\hline$>82$ & 26 & 48 \\
\hline
\end{tabular}

Note. Median values were used as cut-off for statistical analysis.

(Gomez-Iturriaga et al., 2015). The cumulative incidence of pain flare ranged between 23\%-68\% (Chiang et al., 2013; Pan et al., 2014) when patients were treated for a spinal metastases and was $10 \%$ for extraspinal sites (Owen et al., 2014). According to current literature, known predictors of pain flare onset are single-fraction dose regimen (Pan et al., 2014) and lack of steroid pretreatment (Khan et al., 2015). Interestingly, no differences in terms of incidence of pain flare were found between patient groups, most notably for whom it concerns dose schedule and prior administration of steroid premedication. In particular, steroid administration before SBRT is based on clinician's judgement in our Institution and not systematically performed: This might explain more frequent use of steroids in patients receiving opioids, whose pain control might appear frailer and influence clinical decision. Little is known about the physiopathology of pain flare, though the most accepted mechanism is radiation-induced inflammation with consequent oedema of the periosteum (Svendsen et al., 2005): For this reason, the mean and maximum dose delivered to the target lesion and to the planning treatment volume was collected for all patients. Since substantial heterogeneity existed among different dose schedules, dose parameters were recalculated in equivalent $2 \mathrm{~Gy}$ doses to allow for comparison. After careful comparison of dose/volume parameters and incidence of pain flare, no correlation was found, suggesting that more complex mechanisms than linear dose relationship might underlie the development of pain flare: More baseline radiobiological studies are probably needed to shed light on the dosimetric determinants of this condition and propose dosesparing strategies. No other toxicities were reported following SBRT; however, longer follow-up might be needed to confirm the absence of chronic complications such as myelopathy and fractures.

We also focused on the subset of patients who presented with active pain at baseline evaluation to further characterise their conditions and to identify predictors of impaired pain control. As expected, only the size of the target lesion was correlated with the probability to develop pain. In addition to patients and treatmentderived variables, we investigated the use and prognostic value of the ECS-CP, a validated tool developed to study pain complexity in cancer patients and, consequently, difficulty in achieving adequate analgesia. Four out of five items that compose the tool were correctly identified in our cohort; only cognitive impairment was not found in any patients, probably due to scarce compliance of those patients to the stringent requirements of SBRT that resulted in selection of patients with preserved cognitive function. Conversely, addiction to alcohol and cannabis was found in one case. Incident pain $(n=8)$ and neuropathic component $(n=6)$ were the most frequently identified complexity indicators, while psychological distress linked to pain-related aspects of the disease was assessed in four patients. Interestingly, the presence of $\geq 2$ ECS-CP complexity indicators was correlated to shorter pain control, resulting in a time to pain progression of 4 months vs. 30 months. There is a lack of data concerning the applicability of the ECS-CP tool in the pain outcome after radiotherapy to painful metastases. However, if further assessment is warranted in larger prospective cohorts, the ECS-CP may prove useful to stratify patients in function of the expected benefit from radiotherapy and to identify other critical areas of intervention (such as pharmacological/neurosurgical management of neuropathic pain, early diagnosis and counselling for psychological distress, and management of addictive behaviours) in order to overcome pain complexity and obtain adequate analgesia.

Pain control at 1 year was maintained in $62 \%$ of patients with active pain prior to SBRT. Data from literature show pain control in $27 \%-100 \%$ of patients at 6 months (Chang, Youn, Park, \& Rhee, 2009; Gibbs \& Radiosurgery, 2003; Wang et al., 2012) and 54\%-85\% (Nguyen et al., 2010; Sheehan et al., 2009) at 1 year. However, definition of pain control was inconsistent among different studies or was not clearly distinguished from pain response: For this reason, we have chosen the more stringent definition used by Chow et al. (2005). Moreover, in the majority of cases, pain control evaluation was carried out in the whole treated population and not only in the subgroup with baseline pain. Future prospective studies should agree on common definition for this endpoint and selection of the target population, in order to allow for interstudy comparison. It 

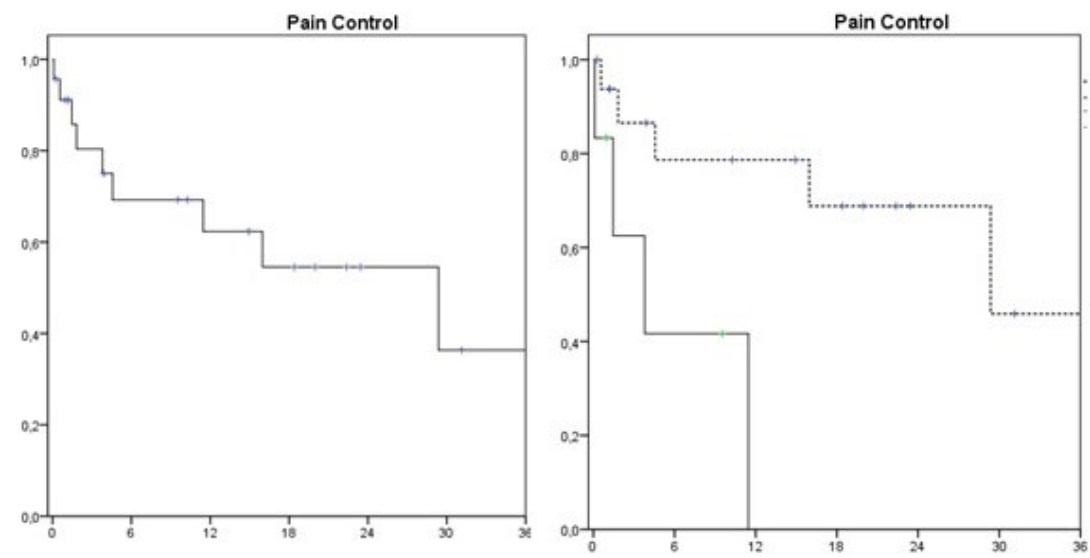

FIG URE 1 Kaplan-Meier plot of pain control in painful patients $(n=23)$. Left: overall pain control. Right: pain control in presence of $\geq 2$ ECS-CP indicators (solid line) vs. $<2$ ECS-CP indicators (dashed line) $p=0.012$

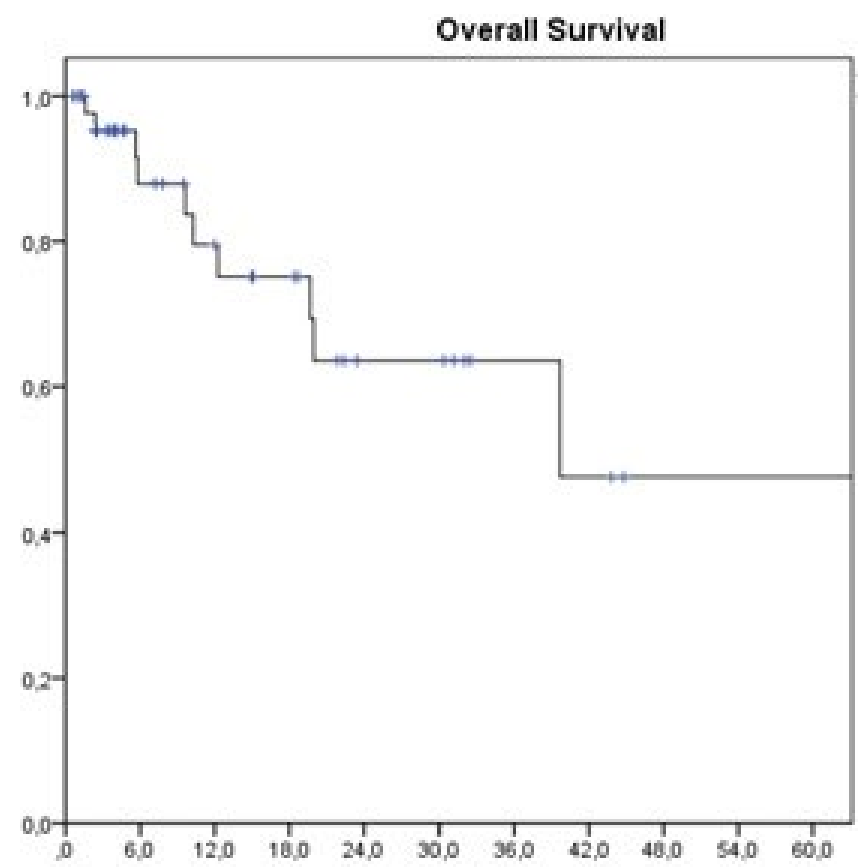

FIGURE 2 Kaplan-Meier plot of overall survival in patients treated with SBRT for bone metastases $(n=48)$

is noteworthy that, in our cohort, median survival after SBRT was 39 months, with only $12 \%$ of patients deceasing within 6 months of the treatment. Due to expected long survival, it is mandatory to obtain durable pain control in metastatic patients and to consider multidisciplinary management to achieve this goal, even in case of treatment failure (Mercadante \& Fulfaro, 2007):For example, surgical rescue was possible in two patients with painful bone progression after SBRT.

There are several limitations in our study. In first instance, its retrospective design with subsequent risk of bias due to data loss, lack of control on confounders and possible under-report of addictive behaviours. Secondarily the small sample size did not allow for further subset analysis. This could be particularly useful in patients with complex pain profile who might deserve further characterisation according to the predominant ECS-CP feature in order to better assess areas of therapeutic intervention. Finally, due to technical complexity of SBRT compared to palliative RT, a selection bias may have occurred resulting in the indication to SBRT restricted only to compliant, cognitively non-impaired patients. To investigate pain treatment outcome after SBRT and the possible predictive role of tools such as ECS-CP, future studies need to be conducted prospectively, enrolling larger samples of patients for an adequate follow-up time with clear identification of predictive factors and combined use of other pain management modalities.

\section{5 | CONCLUSIONS}

In patients affected by bone metastases treated by SBRT, pain flare was observed in $38 \%$ of cases irrespectively of steroid premedication and dose regimen. In patient with active pain at baseline, pain control was obtained in $62 \%$ of patients at 1 year. Presence of $\geq 2$ complexity indicators at the ECS-CP assessment was correlated with impaired pain control and may deserve future investigation in prospective studies.

\section{ACKNOWLEDGEMENT}

ND Klass has received grants from the Swiss Cancer League. We thank them for the generous funding.

\section{ORCID}

Mauro Loi iD http://orcid.org/0000-0002-0360-3275

\section{REFERENCES}

Benedict, S. H., Yenice, K. M., Followill, D., Galvin, J. M., Hinson, W., Kavanagh, B., ... Yin, F. F. (2010). Stereotactic body radiation therapy: The report of AAPM Task Group 101. Medical Physics, 37, 4078-4101. 
Bhattacharya, I. S., \& Hoskin, P. J. (2015). Stereotactic body radiotherapy for spinal and bone metastases. Clinical Oncology, 27, 298-306.

Chang, U. K., Youn, S. M., Park, S. Q., \& Rhee, C. H. (2009). Clinical results of Cyberknife radiosurgery for spinal metastases. Journal of Korean Neurosurgical Society, 46, 538-544.

Chiang, A., Zeng, L., Zhang, L., Lochray, F., Korol, R., Loblaw, A., ... Sahgal, A. (2013). Pain flare is a common adverse event in steroid-naïve patients after spine stereotactic body radiation therapy: A prospective clinical trial. International Journal of Radiation Oncology, Biology and Physics, 86, 638-642.

Chow, E., Hoskin, P., Mitera, G., Zeng, L., Lutz, S., Roos, D., ... International Bone Metastases Consensus Working Party (2012). Update of the international consensus on palliative radiotherapy endpoints for future clinical trials in bone metastases. International Journal of Radiation Oncology, Biology and Physics, 82, 1730-1737.

Chow, E., Ling, A., Davis, L., Panzarella, T., \& Danjoux, C. (2005). Pain flare following external beam radiotherapy and meaningful change in pain scores in the treatment of bone metastases. Radiotherapy and Oncology, 75, 64-69.

Cox, B. W., Spratt, D. E., Lovelock, M., Bilsky, M. H., Lis, E., Ryu, S., ... Yamada, Y. (2012). International Spine Radiosurgery Consortium consensus guidelines for target volume definition in spinal stereotactic radiosurgery. International Journal of Radiation Oncology, Biology and Physics, 83, e597-e605.

De Bari, B., Alongi, F., Mortellaro, G., Mazzola, R., Schiappacasse, L., \& Guckenberger, M. (2016). Spinal metastases: Is stereotactic body radiation therapy supported by evidences? Critical Reviews in Oncology/Hematology, 98, 147-158. https://doi.org/10.1016/j. critrevonc.2015.11.002

Fainsinger, R. L., Nekolaichuk, C. L., Lawlor, P. G., Neumann, C. M., Hanson, J., \& Vigano, A. (2005). A multicenter study of the revised Edmonton Staging System for classifying cancer pain in advanced cancer patients. Journal of Pain and Symptom Management, 29, 224-237.

Gibbs, I. C., \& Chang, S. D. (2003). Radiosurgery and radiotherapy for sacral tumors. Neurosurgical Focus, 15, 1-5.

Gomez-Iturriaga, A., Cacicedo, J., Navarro, A., Morillo, V., Willisch, P., Carvajal, C., ... Bilbao, P. (2015). Incidence of pain flare following palliative radiotherapy for symptomatic bone metastases: Multicenter prospective observational study. BMC Palliative Care, 14, 48.

Hird, A., Wong, R., Flynn, C., Hadi, S., deSa, E., Zhang, L., ... Chow, E. (2009). Impact of pain flare on patients treated with palliative radiotherapy for symptomatic bone metastases. Journal of Pain Management, 2, 401-406.

Hong, J. C., Ayala-Peacock, D. N., Lee, J., ... J. K. (2018). Classification for long-term survival in oligometastatic patients treated with ablative radiotherapy: A multi-institutional pooled analysis. PLoS One, 13(4), e0195149.

Jacobson, A. F., Shapiro, C. L., Van den Abbeele, A. D., \& Kaplan, W. D. (2001). Prognostic significance of the number of bone scan abnormalities at the time of initial bone metastatic recurrence in breast carcinoma. Cancer, 91, 17-24.

Khan, L., Chiang, A., Zhang, L., Thibault, I., Bedard, G., Wong, E., ... Sahgal, A. (2015). Prophylactic dexamethasone effectively reduces the incidence of pain flare following spine stereotactic body radiotherapy (SBRT): A prospective observational study. Supportive Care in Cancer, 23, 2937-2943.
Loblaw, D., Wu, J., Panzarella, K. P., \& T, Smith K, Aslanidis J, Warde P, (2007). Pain flare in patients with bone metastases after palliative radiotherapy-a nested randomized control trial. Supportive Care in Cancer, 15, 451-455.

Mercadante, S., \& Fulfaro, F. (2007). Management of painful bone metastases. Current Opinion in Oncology, 19, 308-314.

Nguyen, Q. N., Shiu, A. S., Rhines, L. D., Wang, H., Allen, P. K., Wang, X. S., \& Chang, E. L. (2010). Management of spinal metastases from renal cell carcinoma using stereotactic body radiotherapy. International Journal of Radiation Oncology, Biology and Physics, 76, 1185-1192.

Owen, D., Laack, N. N., Mayo, C. S., Garces, Y. I., Park, S. S., Bauer, H. J., ... Olivier, K. R. (2014). Outcomes and toxicities of stereotactic body radiation therapy for non-spine bone oligometastases. Practical Radiation Oncology, 4, e143-e149.

Paice, J. A., \& Cohen, F. L. (1997). Validity of a verbally administered numeric rating scale to measure cancer pain intensity. Cancer Nursing, 20, 88-93.

Pan, H. Y., Allen, P. K., Wang, X. S., Chang, E. L., Rhines, L. D., Tatsui, C. E., ... Ghia, A. J. (2014). Incidence and predictive factors of pain flare after spine stereotactic body radiation therapy: Secondary analysis of phase 1/2 trials. International Journal of Radiation Oncology, Biology and Physics, 90, 870-876.

Sheehan, J. P., Shaffrey, C. I., Schlesinger, D., Williams, B. J., Arlet, V., \& Larner, J. (2009). Radiosurgery in the treatment of spinal metastases: Tumor control, survival, and quality of life after helical tomotherapy. Neurosurgery, 65, 1052-1061.

Svendsen, K. B., Andersen, S., Arnason, S., Arnér, S., Breivik, H., Heiskanen, T., ... Jensen, T. S. (2005). Breakthrough pain in malignant and non-malignant diseases: A review of prevalence, characteristics and mechanisms. European Journal of Pain, 9, 195-206. https://doi. org/10.1016/j.ejpain.2004.06.001

Van Oorschot, B., Rades, D., Schulze, W., Beckmann, G., \& Feyer, P. (2011). Palliative radiotherapy-new approaches. Seminars in Oncology, 38, 443-449.

Wang, X. S., Rhines, L. D., Shiu, A. S., Yang, J. N., Selek, U., Gning, I., ... Chang, E. L. (2012). Stereotactic body radiation therapy for management of spinal metastases in patients without spinal cord compression: A phase 1-2 trial. Lancet Oncology, 13, 395-402.

WHO's Pain Ladder (1986). https://www.who.int/cancer/palliative/ painladder/en/

\section{SUPPORTING INFORMATION}

Additional supporting information may be found online in the Supporting Information section at the end of the article.

How to cite this article: Loi M, Klass ND, De Vries KC, et al. Pain flare, complexity and analgesia in bone oligometastases treated with stereotactic body radiation therapy. Eur J Cancer Care. 2018;e12915. https://doi.org/10.1111/ecc.12915 